\title{
Chapter 4 \\ The Process of Internalisation. Theoretical and Practical Implications of the Study on the Phases of the Development of Mental Actions
}

\section{Outline of Lecture 4}

In this lecture, Galperin gives exhaustive descriptions of the phases or forms of learners' activities in the process of the development of mental actions. The second phase is the formation of the orienting basis of an action, which reveals the objective features of the action. Creating the orienting basis of the action is achieved by developing a generalised scheme as described in detail in lecture 3. By applying the scheme as a guiding tool, a person who has never been previously exposed to the task completes it one step at a time. A learner's ability to complete the task correctly on multiple occasions can indicate that the scheme of the orienting basis of the action is complete. The orienting schemecan be created by the teacher and offered to learners for them to use. Because it includes a complete set of the features of the target concept, it provides new opportunities for learning. By using this scheme, learners are able to solve various tasks, and the process creates a specific attitude toward learning: mastering the target concept becomes a mean for achieving the personal success of each individual learner. The set of features included in the generalised scheme can not only be offered to learners but also constructed by learners under the guidance of a teacher by solving problems that arise from the contradiction of facts. Moving step-by-step under the guidance of a teacher, learners identify the characteristic features of the target concept and, in doing so, create a complete scheme of the orienting basis of the action. When the scheme of the orienting basis has been created, it can be applied by learners to solve various problems.

In the third phase, the materialised form of an action, learners engage in and interact with material or materialised objects that carry the meanings encapsulated in these objects. Materialised action is one of the most important forms because the full composition of the action can be shown and transferring the initial materials into desired outcomes can be demonstrated. This form of action can be used in the process of re-teaching when the action developed with learners does not match the requirements of the task. In the materialised form, the action can be fully deployed 
and slowed down so that its every step can be traced by the learners. In summary, the desired properties of the action can be developed in the materialised form of the action. When the action starts to flow smoothly in the learners, it is time to transfer to the next phase or form of the action. This is achieved by removing the material or materialised support, and learners perform the action in the form of externalised social speech (communicated thinking).

In the phase of communicated thinking, for the first time, the action takes the form of an ideal action performed with images of the material or materialised objects. Learners' speech should be framed in a publicly objective form to be understood by other people. Learners do not talk about the action, but they complete the action through talking. When the action in the form of social speech (communicated thinking) systematically demonstrates accuracy and the required speed, the action is transferred to the fifth phase, which is silent external speech (dialogical thinking).

In the phase of dialogical thinking, by visualising the material or materialised objects that the learners interacted with previously, the action is performed by silent speech or talking to oneself. Finally, in the sixth phase, the action is performed in hidden speech, which Galperin refers to as acting mentally. In this phase, artificial fragmentation into individual units is suspended, and the action acquires its natural flow. In this phase, the maximum automation of the action can be achieved.

Galperin points out that some phases or forms of the action can be rearranged or restructured. However, he recommends following the suggested sequence of the phases particularly when a new concept is introduced. He argues that automation does not hinder creativity, and by achieving the automation of actions, learners develop ownership of their actions. When maximum automation is achieved, learners do not interact with the objects but with the meanings of the words that describe these objects. External social speech is transferred to the mental plane, and silent speech is used. Eventually, silent speech is substituted by meanings, and the action can be performed quickly and automatically.

Finally, Galperin elaborates on the method of psychological research as a method for studying the development of psychological phenomena with the required properties. He does not reject the method of self-observation, which can be used to explore a mental phenomenon. However, to examine the phenomenon, Galperin argues that the phenomenon must be created. He continues his argument by criticising the notion of epiphenomenalism or mechanical materialism - explaining that it is in the work of the brain that one should search for mechanisms of the human mind and consciousness. However, to develop human consciousness, a system of opportunities should be created by designing external and internal human activities. Galperin points out that the phases of the development of mental actions evolved during 20 years of research and considerable effort was needed to identify them. Moreover, in learning, the phases may not occur in a sequential order. If learners are exposed to a completely new task or concept, then the action aimed at developing this concept should be fully deployed and explained by following the suggested phases. However, even then, the phases may overlap, and contractions across the phases may occur. When learners begin to move from a fully deployed process to a contracted one, such an action may resemble traditional teaching: there is an explanation of a new task, then there 
is an external material action, and then everything happens as if by itself. However, if learners have been exposed to the phases of the development of mental actions, they will be able to control their learning. They are able to do so because they have transferred the action from the external to the internal plane and turned it into an individual achievement. By creating and performing future mental actions on the external plane, many more tasks may become achievable for learners. For example, based on the suggested system, many topics studied in grades five and six could be learned by children in the first grade and sometimes even by older pre-schoolers. What once needed to be understood in words that were often unsaid, is now presented as an objective reality with clearly visible links and relationships. By applying the phases of the development of mental actions, the alignment in the performance of many learners can be achieved. This alignment eliminates the problem of variations in performance and achievements within a class, opening up another approach to mass education. At the end of the lecture, Galperin discusses the psychological grounds of achieving and underachieving students. He points out that underachieving students do not need extra time for tuition, but they do need specific interventions that are aimed at compensating missed phases of the actions that students need to master.

\section{Lecture 4}

In the previous lecture we started talking about the final, the third, subsystem of the development of mental actions- the subsystem that transfers an originally external action to the inner plane of the learner. I told you that we can distinguish six consecutive phases, and I gave a detailed explanation of the first one.

The first phase is the formation of the motivational basis of the action. We often neglect this aspect, but motivation of one type or another is always present. However, if motivation remains neglected, learning may not even happen.

Now we turn to the second phase. The second phase is creating a scheme of the orienting basis of the action. I have already told you about this scheme when talking about the system of conditions for performing new actions. By following the scheme as a guiding tool, a person who has never been previously exposed to a task, completes it by moving one step at a time. What is important is that a person completes the task correctly, and not once, by chance, but many times, achieving a correct result every time. A learner's ability to complete the task correctly on multiple occasions can indicate that the scheme of the orienting basis of the action is complete.

I have already told you what the scheme consists of and that how it is presented to the learner is important. Without more elaboration, I shall only say that the way tasks are usually formulated is far from being complete in relation to all the requirements needed for a fully-fledged action.

The orienting scheme can be created by the teacher and offered to a learner for him to use. Since the scheme is a complete set of features, it provides new opportunities for learning. 
This brings us to a point that has not been given enough attention in the past. The point is that the scheme of the orienting basis of the action displays the objective features of an action and, therefore, it is only a starting point for the action a learner will engage in. As a consequence of using this scheme the entire learning process shifts to being the achievements of the individual participants. The theoretical underpinnings of the orienting basis of the action are provided as a set of features so that a learner does not spend time getting into the details of the theoretical sources but proceeds straight to the action. Naturally, the main focus is how the student moves forward in this action. This focus creates a motivational (and not only motivational) basis for the action. It creates a specific attitude to the target concept when mastering the concept becomes a mean for achieving personal success of a learner.

Finally, there is another way to introduce the scheme of the orienting basis of the action as a complete set of features-when the set of features is not supplied, but rather constructed by the learner, who proceeds from the problems that arise from the contradiction of facts. Moving step-by-step under the guidance of an experimenter or a teacher, the learner creates a complete scheme of the orienting basis of an action. In this case, the solving of a specific task recedes into the background. What comes to the forefront of the learning process is the development of a deeper insight into the target concept, which creates totally new motivation and the teaching-learning process occurs in a completely different way. It is somewhat paradoxical, but it turns out that this initial, orienting phase, comprising a student's own actions is of primary importance. Once the scheme of the orienting basis of an action has been created by a learner, its application does not present any difficulties. Two-thirds of all effort and time is spent on understanding the concept and creating the orientation scheme, but the mastery of the concept itself does not require much work on the part of the learner. There is an essential distinction between the three ${ }^{1}$ fundamentally different ways to create a scheme of the orienting basis of an action, which determine the three types of learning (I'll tell you about them later).

One more remark about the second phase, which is, in fact, the simplest and the most straightforward. One can introduce the orienting scheme by presenting it in the form of notes on a card and then proceed directly to solving problems. You can also introduce the scheme differently by explaining a specific point and writing it on the blackboard, then explaining the second point and, again, writing it on the blackboard. This way is much better because talking and not writing it down is the worst way of introducing new things. However, when you explain something and put it in writing straight away, this creates much more favourable starting points for learning. For example, you can pause your explanation at any time, and then by pointing to what you have already explained, resume it without any difficulty. This creates a particular freedom for the person who explains, and for the person who

\footnotetext{
${ }^{1}$ Galperin introduces the first type of orientation in Lecture 7. He defines it as incomplete, where mediational tools and the essential characteristics of the concept are identified by learners through trial and error. In this case, learning happens slowly with many mistakes and the activity of learning is extremely sensitive to the slightest changes in conditions.
} 
listens to the explanation. This is the second phase-creating the orienting basis of an action.

In the third phase you proceed to solving the problems you selected previously (I told you about this earlier when talking about the third subsystem of the conditions for the formation of mental actions), using the orienting scheme created earlier. A very big question is how this first independent action is performed. Learning a new concept is a new objective process (objective for everyone who comes across it for the first time), and it should be presented in the most objectified form so that it can be observed and traced by a learner very slowly by fully deploying all the phases of the action. This new objective process becomes observable and accessible for learners when they engage with material objects.

Therefore, the first form of learners' independent engagement with a new concept is materialised. Here we should distinguish between two elements: the material object a learner is engaging with, and the orienting scheme a learner follows when performing this action. These are two different and unequal constituent parts of a materialised action and both of them should be initially represented as external material objects.

Of course, it is not always convenient to operate with material objects. They can be either unavailable or inconvenient to use and, actually, they are not always needed in the learning process. A material object can be substituted by a materialised object, which is a transformed form of a material one. You can either use material models or you can use diagrams, representations and even notes. Many think that taking notes is not a material action, in the sense that the record itself does not carry a meaning, but only the signs that you use to make this record. This is not completely true. Psychologically, taking notes is also a materialised action, although a very special one. We label it a materialised action because materialisation of knowledge you learned previously occurs. Just imagine that you engage in algebra by using algebraic notations. You do calculations in writing, which means you perform an action in a materialised form. For example, you have to work with similar terms. You have to find these similar terms, cross them out and replace them with the sum. If you do all this in writing - this is a materialised action. This form of action is very important because in this form you can trace what you have missed out and check if you have solved the problem correctly. The record is a material object that you can turn to later. You can also transfer one part of your record to another record and vice versa.

In summary, the record carries particular meanings (for those who do not understand these meanings, the record does not make any sense at all) and it is a material object. From studies in psychology we know that even what we identify through direct perception, is full of acquired meanings. People perceive the same things differently depending on the premises on which they build their perceptions. In fact, even what is labelled perception is full of publicly accepted meanings. In materialised action, it does not matter what kind of meanings are encapsulated in this record (which is also, of course, essential), but what is important is that these meanings are represented by a material artefact that carries the meaning and which can appear as a real, objective thing. 
So far, we have two forms of representation of an object of an action, and the ways of working with this object—which may be material object and materialised object. In the learning process, it is very important that we can actually substitute the original material objects with all kinds of materialised alternatives, by assigning them the specific and well-defined properties of the objects we are interested in.

The materialised form of action is one of the most important; it is in fact the most important of all the forms, because in this form you can show the full composition of an action, and teach how to transfer the initial material into the desired outcome. This form of action can also be used when we need to re-teach someone if, for example, a person has developed an action that does not match the requirements of the task.

Recently some of my colleagues conducted a study about eye movement in relation to an object. Strict requirements were applied to this movement ${ }^{2}$ : it had to be performed very quickly. It turned out that the eye movement, which we do in everyday situations, such as, clockwise (from left to right) eye tracking around an object, is not suitable for these special, strict requirements. Is not suitable because the eye movement is usually accompanied by lots of vibrations, which a person is unaware of (an eye does it automatically), and because of these vibrations it is impossible to achieve a high and steady speed of eye movement. It turned out that our eyes need to be re-trained to achieve a productive action performed under very strict, predetermined requirements. This is possible by fixing our eyes on a material object, for example, on the edge of a pointer and following the movement of the pointer with our eyes. Only after this kind of training can you get what seems to be a new type of the same movement.

Similar things happen in the army: when a soldier is enrolled, he is taught how to walk. It turns out that if we impose high requirements for an action, we have to re-learn how to do it, because in everyday life we complete many actions in ways which don't meet these high requirements. Hence, one needs to re-learn how to use mechanisms that are hidden within us and over which, it seems, we have no control. You cannot control these mechanisms because you do not know how they work. This control can only be achieved when an object and an action with this object is performed on the external plane. Then you can control your learning! Therefore, the materialised form of the action is the most important form for learning and teaching and especially for any type of re-learning.

However, something else has to be accounted for if the materialised form of action is to satisfy all the requirements. First, it is necessary to fully deploy the action and slow it down so that every step can be traced by the learner. For example, in the process of learning a foreign language a person is given an example of phonetically correct pronunciation, which he tries to imitate, thinking that he is doing it correctly. Actually, most probably he is doing it wrongly because it is not enough to give an example, you have to record immediately what the person has pronounced (by, for example, using a recorder) and then replay it.

\footnotetext{
${ }^{2}$ Podolsky (1973) Experimental formation of visual recognition of objects. Moscow: Moscow State University.
} 
An action should be performed in a form in which it can be easily traced by the learner: in slow motion, deployed and unfolded in such a way that it can be followed by an external observer. In this form, the action can be subjected to what I have already mentioned: differentiation and awareness of this action. These are accomplished by performing the action in the form of speech, which should accompany the action in all its units. With time (if this is possible) a deployed action should be shortened i.e. be accomplished more rapidly. In brief, in the materialised action the desired properties of an action are formed.

Now the question arises: when do we finish this phase and transfer to the next one? After all, this is just a phase, and it is very dangerous to linger at it, without moving ahead. I have already said that automation at any phase indicates its completion. When this happens, we should transfer to the next, higher forms of action. Automation means that the action starts flowing smoothly, accurately and at a sufficiently high speed. When the action reaches these indicators, we begin to remove the material support. However, what material support can be removed? First of all, you can remove the orienting card. When you notice that the learner is looking at the card occasionally or working without using it, you flip the card onto the reverse side. You can also tell the learner that if he forgets anything, he can always look at the card to complete the action, however, after the card has been used, it should be placed with its face down again. This situation encourages the learner not to use the card every time and creates a situation of involuntary memorising. However, if a learner spends too much time using the card, you will get the opposite effect. This is very important because the same tool can perform completely opposite functions. If you overdo the availability of the card as a resource, then the learner might get an impression that there is a memo or reminder which can be used any time, so there is no need to remember things. If I, for example, have an address book, I'm not going to memorise all the addresses; I will turn to the address book whenever I need it. This creates a psychological situation that encourages a learner not to make any effort at remembering things. Many attempts to introduce such memo cards were unsuccessful in the history of different education systems. Therefore, the orienting card should be removed when the time is right.

Later, when the action is transferred to the mental plane, then the initial material should be also removed. Well, let us say, if you want to perform calculations or analysis of any kind, you have to understand not only the formula or the algorithm of the analyses, but also that the initial data or analysed material can be at some point removed and the action will be completed without referring to them. However, this is not so important. What is essential here is the orienting scheme of action itself.

When you remove the material support, the action will not be immediately transferred to the mental plane. First, it is transferred to the plane of externalised social speech, which means that the analysis of this material and the actions with this material will be completed in the form of social speech. This speech is aloud, not only in its form but more importantly in its function as speech directed to another person. It is important that this is both a verbal action, and a message about the performed action. 
The message about the performed action is of crucial importance, because anyone who has worked with people knows that quite often people say the right things; but at the same time, when performing the action, they omit important points, or even say something that is not related to the action, this is a mismatch of action and speech. This mismatch is a very dangerous thing. Critical responses from another person are crucial here because at this point, for the first time, the speech needs to be comprehensive and clear enough for another person to understand it; it is a requirement of its social (public) qualities. This means that a learner has to talk about the action and not only in a way that seems clear to him. A learner can visualise the material object and keep this object in his mind when talking about it. However, the material object is not present, we have already removed it. At the same time, the learner may think that he is doing everything properly. Perhaps he is acting correctly, but speaking about it unclearly. He should learn to speak in a way that is clear enough for another person to understand. Hence, the main emphasis is on the social aspects of speech: the action for the first time acquires the form that exists in the public consciousness. This is so because in this phase the material object is not present, but only its verbal expression: the communicative action acquires the form, which reflects the action with the material object. In the form of externalised social speech the action carries only one possible meaning which is a publicly accepted meaning.

When a learner is acting with material objects, it might seem that speech is of secondary importance. One has to do the job first: here is the initial material; here is the order of the actions; and a final outcome has to be created. This is the main, primary point. When the learner is making sense of the orienting card, speaking, being corrected-all this seems to be of secondary importance. However, in externalised social speech-communicated thinking - there is no material and no cards. Everything in expressed in speech and speech, as a fully-fledged representation of the action, performs its publicly objective function.

This is very important because for the first time the action takes the form of an objective thought. Therefore, logically (not psychologically), an action expressed in speech, is, actually, a thought. On the external plane, when a person moves from action with material objects to communicative action, a transformation from the action with objects to thinking about this action occurs and the person has to recognise that his speech should be framed in a publicly objective form.

However, one should avoidexpecting learners to use definitions, because by doing so, they may access an abstract formula, which does not yet carry the correct meaning for them. Therefore, to begin with, a learner should use his own extensive vocabulary and a teacher should insist on his doing so. Afterwards, when all these different ways of expressing the same thought have been played with, one can frame the speech with a given formula. ${ }^{3}$ Only then will this formula encapsulate a whole variety of speech expressions.

In this phase, the action, now in the form of speech, is applied to solving the same set of different types of tasks as in the previous phase of materialised action. When the action in the form of speech, applied to the different types of tasks, begins to

\footnotetext{
${ }^{3} \mathrm{~A}$ formula may be understood as a definition.
} 
systematically demonstrate its accuracy and the required speed, we can move to the next, the fourth phase - the transfer of the action to the mental plane. At this point an interesting thing happens. Once the action is transferred from the external to the internal plane, you immediately lose control over it. After all, you do not know what the learner is doing mentally. This is a big challenge, which we have only solved to some extent, though a rather important aspect.

We have found a way to control the action that has already been transferred to the mental plane. After all, the orienting card contains numbered instructions. When we transfer to this new form of the action, we can say to the learner: "Now we will work in a new way, I'll either point out a number to you, or you will tell me the number of the instruction you are working on at the moment". The action is performed mentally, only the result of the performed action is announced out loud. In doing so, we monitor the action in its individual operations. However, within individual operations the action happens on the mental plane, which we still do not know how to control. Nevertheless, we do not allow the learner to skip all the operations right away and simply present the final outcome, although very often a person is capable of doing it. Sometimes a learner gives the correct answer, and you realise that this answer includes performing not one, but several operations. In this case you say, "No, do not do it this way yet. First, give me the first answer then the second answer, etc." Of course, we know which answer should be given at each of these operations and if after the final operation has been completed the answer is the correct, we say, "Yes."

This is how we perform operational monitoring. What kind of form does the action take? Is it the first mental action? What does the learner transfer there? He transfers the action that previously was the action in a form of an external socialised speech to the internal plane. In this phase a learner can imagine the material objects or the orienting card, but this is not so important and $\mathrm{we}^{4}$ do not interfere in this process. We require only one thing: that the action is performed as a sequence of operations recorded by voice or symbols, and we get an answer. Therefore, we believe that the first form of the mental action is the action performed in external speech when the learner talks to "himself" silently. This is the most frequently deployed form of speech, without saying anything out loud, not even in a whisper, because a whisper is a form of audible speech and is quite common with children and uneducated people. This form of action comprises the fifth phase-the action in silent external speech.

Once again, we apply this new form of action to the whole range of tasks which we discussed in the previous lecture. When the learner achieves fast and correct completion of every operation of the action, we move to the last, the sixth phase. We say to the learner: "Now we are going to work in the following way: I will give you a problem and you will give me the final answer". The final answer is not given at the end of each operation, instead what we need is the very final answer! In doing so, we remove this artificial fragmentation into individual operations that we had demanded previously, and instead permit an action its natural flow on the inner, mental plane. In this case, the developed action relies on the same content in the

\footnotetext{
${ }^{4}$ Researchers/teachers.
} 
phase of a materialised action, and in the phase of communicative thinking, and in the phase of external silent speech. That is why the action is smoothly following the "tracks" and can be automated easily. We would like the learner to achieve maximum automation of the action in its final form. In order to do so, we require the learners to apply the action in its final form to the whole range of problems.

The action has to be performed correctly with maximum automation, and maximum speed. The slightest mistake - and we must return to the previous phase: either to the phase of externalised social speech (communicative thinking) or even to the phase of a materialised action. Errors are not allowed.

The more we explore this, the more we become convinced that any fluctuations in the performance of actions are not welcome; sometimes some operations can be rearranged or restructured, but you have to follow a certain order, one way or another. These fluctuations are not useful as they can hinder automation and an action that at first may seem to be satisfactory, begins to break down.

Many oppose automation, saying that it supposedly inhibits creativity; but these are different things. Creativity is an ability to solve a new problem. However, some aspects of the solutions to new problems must be already mastered. The best evidence for this is, for example, artistic performance. You know that no actor can achieve a good, creative performance, if he does not bring the technique of his action to maximum automation. Once your technique has been automated, only then do you first achieve the ownership of your actions. This happens if you have ensured sufficient generalisation of the action. When you have not, you should not blame the automation, but blame yourself because you did not form this property of the action.

When maximum automation is achieved, the following happens. Starting with the phase of externalised social speech (communicative thinking) a person is not acting with objects but with the meanings of the words, which express these objects. When externalised social speech is transferred to the mental plane, first externally deployed and precise silent speech is used. However, the more we master this silent speech, the more it becomes an obstacle, because it creates a barrier between individual elements, and, hence, creates a delay. Since the action has been automated, any delay is unnecessary. Something happens that was previously observed only in some intermediate operations: whole elements of the speech action disappear. Speech, being an obstacle for the flow of thoughts, begins to contract. First inner speech appears (that is, only the elements of speech, which reflect the difficult parts of the action and these elements should be deployed so that they can be traced), and everything else gets contracted. This is what is labelled inner speech. However, this is also only an intermediate form between the external silent speech and the form of the action that no longer contains any speech. With time, images of words, articulatory elements of speech begin to disappear from consciousness, because being fragmented they delay the action. What remains is the action comprising the movement of meanings - and scientists have not yet agreed on what a meaning is. Therefore, in the last phase of the action there emerges a phenomenon that previously has been labelled "a pure thought". This means if a person performing an action would like to trace the inner process of solving a problem, he is not able to see anything there, except for a special state labelled as "a state of consciousness". In short, something very vague. 
When "the state of consciousness" was first discovered, it fascinated some psychologists, while others got outraged because they saw this as a proof of idealism and said, "This pure thought, is an experimental proof of idealism". While others, also idealists, but supporters of sensationalism (those who claim that everything in our mind comes from our senses), disagreed, saying: "This is impossible".

Well, they kept repeating: "impossible, impossible", but it still exists. An English scientist conducted a very simple experiment, which clearly demonstrated the existence of a pure thought. The experiment was as follows. A person was sitting at the table that had two buttons. An experimenter said: "I am going to say a word. If you understand the word, press the right button. If you visualise the image corresponding to what you have understood, press the left button. That is all". The person was given very simple, everyday words, such as a glass, a pencil, etc. If the person had understood the word, he pressed the right button and if he had visualised the image corresponding to the word object, he pressed the left button.

It turned out that in such experiments our understanding is significantly ahead of our visualisations. Sometimes we visualise the object and sometimes this does not happen at all, because it is simply unnecessary. For example, if I say: a glass, you understand what I am talking about. Do you really need to imagine a glass? It is not necessary. So, what is our understanding? Neither images nor speech elements are used, but understanding is present. As I have already said, the idea of a pure thought outraged some psychologists and fascinated others. The dispute between these two groups lasted until everyone had had enough, as often happens in psychology. However, the issue had not been resolved and it cannot be solved if you do not know how it occurs. You can understand that pure thought happens only when the automated action is created by you, starting from its original external forms.

So, the final form of a mental action is labelled pure thought when it is, in fact, hidden speech. This form is constructed from the elements of speech present in our consciousness in the form of meanings. Their material shell, including kinaesthetic sensations, articulation and sound images - all this becomes a nuisance and is eliminated. In fact, all these aspects are present, but hidden from view, because meanings belong to words and expressions but meanings are not to be found in the mind. Therefore, the last phase of the action is the action in hidden speech, subjectively expressed as a pure thought.

Now it is extremely important to emphasise the following: we started with the external action with objects, and finished with thinking about this action. When we were tracing this unfolding process, we understood how a new concrete thought was born in its original primary form. There might be secondary forms, which are also hidden, but that is a different matter. What is important is that the initial appearance of a thought is nothing else but a transfer of the action with objects to the human mind and its being processed there, everything that K. Marx had told us.

Therefore, if you study the formation of the action in its final form and trace the unfolding all the way from the material to the ideal action, then you might develop an understanding of how an exquisite psychological phenomenon such as an individual thought is formed. This is very important, because it opens up a new direction for the studying of mental processes. Up until now all mental processes 
have been considered internal and can be studied only by self-observation. This considerably restricts research on these processes. When observing psychological phenomena only in their final form, researchers would make a gesture of helplessness: what can we do, what are we to study?

Of course you can only explore physiological processes; they are material. We are a long way from being able to trace a trajectory of a psychological process in the brain but, in principle, this is possible. However, if we assume that any psychological process is purely subjective, then it becomes impossible to investigate the process.

That is why there is such a strong tendency in modern psychology to find the physiological origins of psychological processes. It is not worth arguing against this tendency, and we are not going to do so, because this will only be relevant, if the physiological origins are revealed. On the other hand, even if these physiological origins are disclosed, they will not explain the psychological processes that are underpinned by these physiological features.

Another direction for research is through examining logic, a direction which is pursued by the outstanding scholar Jean Piaget. He believes that it is quite legitimate to look for physiological explanations of psychological processes. However, this is not sufficient; physiology does not explain the logic of external processes. Therefore, we can use the logic that is employed as an explanation of other psychological phenomena. Well, where is psychology as a science then? As you can see, from this point of view psychology as a science does not exist. There are only phenomena, but they do not constitute the subject of a science. If you know the process of the formation of a particular psychological phenomenon, for example, a mental action, the creating of an image, etc., then it's a different matter. Then the physiological origins may be useful, because people are not made from air. However, these physiological origins only create premises for the realisation of psychological processes, only premises!

With this understanding, we move on to describing the actual method of researching psychological processes. I have already told you about the subject of psychology, and now we turn to the method of psychological research, which is focused on studying the formation of a psychological phenomenon with its necessary properties. If you do not specify a priori these properties, then you will never understand why the phenomenon has turned out in one way rather than another. You can select these properties based on the ideas we were talking about and create the conditions that will allow you to form these properties. When you understand this system then you will be able to recognise why an action is lacking some of the necessary properties (if there really is a lack): not because a person is not capable, but because they were not provided with the conditions for mastering some of the tools of the mental activity.

Up until now, psychology has held the simplified view that a mental phenomenon is a spiritual act that is indivisible into its constituent parts and that it can be either good or bad because of its nature. In fact, a spiritual act is nothing but an action with objects transferred to the ideal plane. This phenomenon may contain specific conceptual tools, which can be applied, mastered and generalised in one way or another, but we have to understand that a mental phenomenon like this is a real process. Transformation of this process to the ideal plane-is also a real process and 
if you do not ensure this transformation, then you might end up with "limited mental activity".

Mental activity has to be understood quite pragmatically. It is a type of work, like any other work performed by people. This work has to be mastered and resourced by proper tools.

Some people think that a mental action is an action of the brain. These views are the relics of old ideas about mental actions, seeing them as existing independently of the real world and therefore not subjected to any restrictions. However, it turns out that mental actions can be resourced with tools that can significantly improve them.

To sum up, our first conclusion concerns the method of psychological research. We do not dismiss the method of self-observation, we use it and accept it. Selfobservation can describe a mental phenomenon. However, this is only a description of the phenomenon and nothing else! What lies behind this phenomenon? We can only discover this when we are creating the phenomenon and shaping it in a controlled process. Then we know what lies behind it. There are no reasons not to take the data of self-observations into consideration; however, to limit us to self-observations means to deny psychology as a science, because science cannot be framed by phenomena only. Once we know all the mechanisms that lie behind these phenomena, then we can begin to understand their role. Philosophers claim that phenomena are important because if you have a complex process, which is hidden from view, then with the help of a phenomenon you can control the whole process. At that point you begin to recognise how useful the phenomenon of mental, contracted and automated action actually is. This is the most profitable, the most economical form of mental activity.

Therefore, a phenomenon-is a special form of very economical work, because you are not dealing with the parts of the process; rather it is presented to you as a sum of the parts and as a phenomenon as a whole. If the phenomenon appears to sit at the top of a pyramid of well-established and thought-through connections, its positive value is greatest.

Most people cannot move beyond the idea that mental phenomena, human consciousness, are in their heads. What lies behind these misconceptions-is their idea of the brain, which is, in fact, a working organ. Their argument is that because psychological phenomena do not manifest themselves in any way, it is in the work of the brain that one should search for mechanisms of human mind and consciousness. However, this is a very subjective point of view. The notion of epiphenomenalism or mechanical materialism is still quite common. This notion claims that the brain produces mind, but mind is useless, because it is an ideal phenomenon. In addition, these misconceptions suggest that ideal notions like thoughts should not affect the material and confuse all the laws of material things.

This view, suggested by Hobbes, still exists. Many physiologists have a similar understanding of the human mind. Well, there is a brain, which is worth studying, but what is the human mind?

Now you understand that a brain and human brain especially-is a system of opportunities. Which of these opportunities will be realised depends on how we construct external and internal human activity. These opportunities cannot be realised 
by the work of the brain by itself. A subject engages in the action, his mind does not act by itself, but a subject as an owner of the mind creates a form of ideal action.

What is the practical significance of the system we are talking about? Do the phases of mental development always have to be in such a fully-fledged form? If it were so, it would not have been so difficult to identify these phases. We, on the other hand, have spent more than 20 years studying these phases. The point is that development does not always follow these clear phases. Why? Just recently A. F. Karpova ${ }^{5}$ defended her dissertation on this subject. Her research has shown that if you deal with new material and a new task, then you need to deploy the full sequence of the phases of the action. If you need to repair a previously formed action, then you should also follow all the phases. However, if you apply the phases of the development of mental actions systematically, you will find that overlaps and contractions across the phases will occur.

For example, speech is a very important form of action. First, students learn to talk, so that another listener can understand both them and the content of the action performed. When a student understands the requirements that are imposed on his speech, he begins to apply these requirements appropriately on other occasions. Therefore, there might be no need to include the phase of external social speech because the learner already knows how to speak in the required way.

The second point in relation to the reduction of the phases of the development of mental actions is that the learners begin to move from a fully-deployed process to a contracted one, shortened in such a way that it seems to last for only a moment. For example, when a learner is introduced to a task, he processes it in his head according to already well-established automatisms. The learner gets the answer at once, without any preliminary work. This marks the transition from successive to simultaneous action.

The third point - is the use of automated actions for solving new problems. This differs from simultaneous action in the way that there is a clear sequence of operations, but they happen very quickly.

If you have been applying the phases systematically to solving problems, then when they are applied to other problems, these phases contract and learning may connect with the form of traditional teaching: there is an explanation of a new task, then there is an external material action, and then everything happens as if by itself.

The initial awkwardness or challenge of the materialised action pays off quickly by: i) the speed of the formation of the action, and ii) the fact that the materialised action may be eliminated from the learning process. An extremely important point is that a learner becomes able to control his learning. After all, what has the process of learning been so far? Memorising. Something is explained to you, then you are asked to memorise it and then you are tested to see if you have done so. Nothing else. How this memorising happens remains undiscovered. When you apply the phases of the development of mental actions, you transfer the external action to the internal

\footnotetext{
${ }^{5}$ Karpova (1977) Changes in the phases of the development of mental actionsin their systematic application. Controlled formation of psychological processes. Edited by P. Ya. Galperin, Moscow: Moscow State University.
} 
mental plane, turning it into the individual achievement of a learner. In addition, we can control this process.

Because we carry out the foundations for creating and performing future mental action on the external plane, many things become much easier and more accessible for learners than in traditional teaching, where everything is presented mainly in words and is only sometimes illustrated.

For example, we discovered that very many of the topics that had been studied in grades five and six could, with the help of our system, be learned by children in the first grade, and sometimes even by older pre-schoolers. It is often suggested that children appear to have hidden abilities, likening them to deep reservoirs of oil which, when revealed, begin to gush.

Well, this is nonsense. They think that the brain is full of hidden abilities and if we drill a hole in the brain, the abilities begin to gush. This is a complete nonsense! The point is that you simplify students' work. What was once needed to be understood from words, and often words, which are largely unsaid, is now presented as an objective reality with its obvious links and relationships. Therefore, the point is not that there are some kinds of abilities which can be revealed, but that the learning activity of a child is organised differently. The point is that new tools can empower a child's mental activity.

Another practical matter is that, due to the simplification of the process, we get a new solution to an old problem - the problem of the alignment of the performance of a large number of learners. Traditional teaching is classroom teaching, and a class is a significant amount of people, selected randomly. Because of that there is a big difference in performance and in abilities.

This issue was first raised back in the late nineteenth century and attempts were made to group all students according to their abilities. Then so-called talent tests appeared, and these tests are still in use, although significantly improved. Indeed, people can be divided into groups according to the level of their development and the differences between these groups can persist for their whole lives. However, what lies behind this grouping - is another matter. In some European countries, the issue is resolved in the following way: students of similar abilities are grouped together, and each of these groups has their own curriculum, their methods of teaching, etc. This approach often has economic and other implications, because you just select people according to pre-defined criteria. One group can easily master the curriculum, another group will require more time on it and the third group will not manage it at all.

It has turned out that the use of the phases of development of mental actions, applied to ordinary children, results in the alignment of their performance. This means that the general education programme appears to be quite accessible for ordinary children. Underachieving students (of course, these children are always present) need additional tuition, but only at the beginning and for a very short period. Then the alignment of students' achievements happens. I would like to emphasise that we are discussing the alignment of performance and achievements, not abilities. We will discuss abilities separately-we do not deny abilities exist; they just need to be explained appropriately. However, in relation to the general curriculum, you can get 
this kind of alignment of performance so that the worst performance would be as different from the best, in the way that $98 \%$ is different from $92 \%$, no more than that. This alignment eliminates the problem of variation in performance and achievements within a class.

These teaching methods open up another approach to mass education. I have already told you that we selected some children at the end of their first year of schooling and examined the development of their mental actions in relation to arithmetic. We found very clear differences, which generally corresponded to the evaluation of the teacher. In fact, the differences were very interesting.

It appeared that the students who used to get a grade two (on the scale 1-5) were those who were stuck at the early phases of actions. In their attempt to catch up with their peers, they automated these earliest phases, because the automation results in the acceleration of the performance of the action. Trying to catch up with their peers, these less successful students automated what they could do and therefore got stuck in these early phases. The trouble is that when the teacher wants to help them, and engages with them, this engagement happens in the same way as the action had been performed previously. The students simply get some additional tasks. At this point, everything depends on the teacher: if we have a good teacher, let us say, a talented teacher, he may notice that a child is not working the way he should. The teacher modifies how the student works and this can lead to a positive outcome. If we have an average teacher, he does his job properly, working extra hours with the less successful student, but he does not notice what is missing in the child's approach. The teacher just spends more time on solving problems, but a child gets completely stuck in the early phases and does not achieve what they should. Then the teacher says: "I have done everything I could, but it's hopeless". Everything stops here. This is the psychology of underachieving, grade two students.

Now let us explore why a child becomes "a grade three student". On one hand, "a grade three student" is learning something and, on the other hand, why doesn't he get a grade four? It turned out, that these children master the first phases of the action, memorise the results of these first phases and skip the intermediate mental phases of the action. Therefore, whenever a learner may rely on the available data, he produces the right answer. If the data is not available, he turns to the expanded materialised action. However, if he needs to do something mentally, he simply cannot do this. Therefore, this student almost always gets a grade three because he can perform some actions on the external plane, but if the action has to be performed mentally, this student is helpless.

Now "grade four students". They are very interesting and colourful people. They differ from "grade five students" by the fact that they always have something unfinished. Sometimes, for example, they may not generalise, or some of the phases of the action have not been automated. In short, they always have some small faults, these faults vary, but they are always present.

What about "grade five students"? These students are those who have mastered all the phases of the development of mental actions. They satisfy the complete set of the requirements. Sometimes, one comes across students who are not genuine "grade five students". When we presented them to the teachers, the teacher agreed that the 
grade five was given as a form of encouragement. This encouragement can be of different kinds; sometimes, a teacher sees that the student deserves a grade four, but he tries his best, makes every attempt, so he is awarded "a five". Somebody can be given "a five" just because "he is just a good boy"; in this case a grade is given for his moral qualities. On the other hand, there are "grade five students" who are given "a four" so that they do not become conceited. In short, these personal relations and considerations are very confusing, and teachers admit their existence.

Now the final, very interesting point. The grades given by a teacher usually summarise many different aspects, but the action of a learner in various phases reflects what kind of learner he is. We chose seven "grade two students", underachievers, and identified what was automated with these students and what skills were missing. The first thing we did was a "de-automation". We did it so that the students were returned to the expanded, played in a slow motion, materialised action. This phase was used as a starting point for the development of the mental operations of the action. Very quickly, within two weeks, these underachieving students were transformed to a completely different level: almost all of them became "grade four students", a few even "grade five students" and one became a "grade three student". However, all of them became achieving students. The point is that underachieving students do not need extra time for tuition, but they need specific interventions, which are aimed at compensating for earlier missed operations of the action that students need to master.

Open Access This chapter is licensed under the terms of the Creative Commons Attribution 4.0 International License (http://creativecommons.org/licenses/by/4.0/), which permits use, sharing, adaptation, distribution and reproduction in any medium or format, as long as you give appropriate credit to the original author(s) and the source, provide a link to the Creative Commons license and indicate if changes were made.

The images or other third party material in this chapter are included in the chapter's Creative Commons license, unless indicated otherwise in a credit line to the material. If material is not included in the chapter's Creative Commons license and your intended use is not permitted by statutory regulation or exceeds the permitted use, you will need to obtain permission directly from the copyright holder.

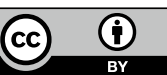

